Nervenarzt 2022 · 93:459-467 https://doi.org/10.1007/s00115-021-01214-w Angenommen: 9. September 2021 Online publiziert: 15 . Oktober 2021 () Der/die Autor(en) 2021

\section{„iFightDepression“ im stationären Setting}

\section{Evaluation eines internetbasierten, therapeutenbegleiteten Selbstmanagementprogramms auf einer Spezialstation für affektive Störungen}

\author{
Julian Schwarz ${ }^{1}$ Nicole Mauche ${ }^{1}$ - Caroline Oehler ${ }^{2}$. Christine Rummel-Kluge ${ }^{1}$. \\ Ulrich Hegerl ${ }^{3} \cdot$ Maria Strauß' $^{1}$ \\ ' Department für Psychische Gesundheit, Klinik und Poliklinik für Psychiatrie und Psychotherapie, \\ Universitätsklinikum Leipzig, AöR, Leipzig, Deutschland \\ ${ }^{2}$ Stiftung Deutsche Depressionshilfe, Leipzig, Deutschland \\ ${ }^{3}$ Senckenberg Professur, Klinik für Psychiatrie, Psychosomatik und Psychotherapie, Goethe-Universität \\ Frankfurt am Main, Frankfurt am Main, Deutschland
}

Hintergrund: E-Mental-Health (EMH) spielt im ambulanten Versorgungssetting depressiver Störungen zunehmend eine Rolle. Ziel dieser Studie war die Implementierung und Evaluierung der Anwendbarkeit und des Nutzens des onlinebasierten Selbstmanagementprogramms „iFightDepression“ (iFD) als Zusatzangebot im Rahmen einer leitliniengerechten Behandlung auf einer Spezialstation für affektive Störungen. Methodik: Es wurden insgesamt 78 stationäre PatientInnen mit einer unipolaren Depression unterschiedlichen Schweregrades (ICD-10 F32.0-3, F33.0-3) oder einer Dysthymie (F34) rekrutiert. Die Interventionsdauer mit dem iFD-Tool belief sich vom Zeitpunkt der stationären Aufnahme bis zur Entlassung und wurde vom Stationspersonal begleitet. Die Erhebung der Symptomschwere sowie von Parametern zur Behandlungserwartung und Therapievorerfahrung erfolgte online vor der Intervention (T0), die Interventionszufriedenheit wurde unmittelbar vor der stationären Entlassung (T1) mittels eines Paper-pencil-Fragebogens erfasst.

Ergebnisse: Von den 78 Teilnehmenden loggten sich 42 mindestens einmal in das iFD-Tool ein. Es zeigten sich moderat hohe Erwartungswerte sowie leicht überdurchschnittliche Zufriedenheitswerte bezüglich der Behandlung. $67 \%$ der aktiven NutzerInnen gaben an, das iFD-Tool poststationär weiter nutzen zu wollen. Wesentliche Gründe gegen die Nutzung waren eine kurze Aufenthaltsdauer, eine schwere depressive Symptomatik und fehlende Medienkompetenz.

Diskussion: Eine Implementierung des iFD-Tools im stationären Setting ist prinzipiell machbar und führte zu positiven Rückmeldungen seitens der Nutzerlnnen. Auch konnten sich die meisten aktiv an der Studie Teilnehmenden eine weitere Nutzung des iFD-Tools nach der Entlassung gut vorstellen. Allerdings weisen die insgesamt geringen Nutzungs- $(42 / 78,54 \%)$ und Komplettierungsraten auf Nutzungsbarrieren hin, deren Überwindung entsprechende Anpassungen der Intervention speziell für den stationären Einsatz erforderlich machen.

\section{Schlüsselwörter}

E-Mental-Health · Depression · IKVT · IFightDepression · Selbstmanagement 
138 PatientInnen wurden zur Teilnahme befragt ( $87 w, 51 \mathrm{~m}$, Alter $M=40.8, S D=10.9$ )

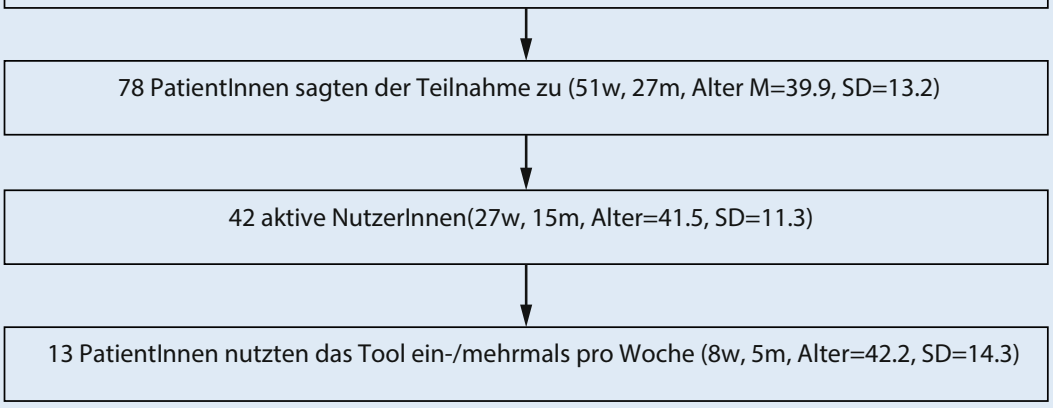

Abb. 1 ॥ Flowchart derTeilnehmenden. $m$ männlich, wweiblich,M Median,SDStandardabweichung

Eine Vielzahl internet- und mobilbasierter Interventionen (IMI) wurde speziell für die Bedürfnisse depressiver PatientInnen entwickelt. Die Prüfung der Wirksamkeit und Anwendungssicherheit erleichtert die Auswahl und Verbreitung dieser Tools.

\section{Hintergrund und Fragestellung}

Depressionen sind eine weit verbreitete und schwerwiegende psychische Störung mit einer Lebenszeitprävalenz von ca. $11,6 \%$ [7]. Trotz der hohen Prävalenzraten gelingt nur $50 \%$ der Betroffenen ein Zugang zu geeigneten Behandlungsangeboten, nur ein Drittel erhält am Ende eine depressionsspezifische Behandlung [24].

Eine Möglichkeit zur Verbesserung der Versorgungssituation besteht in der Nutzung von E-Mental-Health(EMH)-Programmen, welche niedrigschwellig verfügbar, kostengünstig, ortsunabhängig und flexibel in der Anwendung sind [19]. Allerdings werden EMH-Angebote in der Allgemeinbevölkerung generell als weniger hilfreich im Vergleich zu Face-to-face-Therapien wahrgenommen [3], wobei Studien zeigten, dass eine internetbasierte kognitive Verhaltenstherapie (iCBT) ebenso effektiv wie eine Face-to-face-Psychotherapie sein kann [1]. Der positive Effekt von EMHProgrammen verstärkt sich bei therapeutischer Begleitung der Intervention, welche vor allem bei schwergradiger Depression sehr relevant zu sein scheint [14]. Onlineinterventionen eignen sich insbesondere zur Reduktion depressiver Symptome bei leicht- oder mittelgradiger Depression
[27], wobei gezeigt werden konnte, dass begleitete iCBT auch bei Personen mit Symptomen einer schweren depressiven Erkrankung zu einer Reduktion der Symptomatik führen kann [22]. Zur Akzeptanzförderung und Erhöhung der Nutzungsbereitschaft eignen sich besonders Programme, welche textbasierte, expertengestützte Informationen integrieren [4]. Im klinischen Behandlungskontext scheint allerdings die Akzeptanz der PatientInnen für EMH-Therapien im Vergleich zu NutzerInnen, welche sich über das Internet informiert und sich eigenständig für die Nutzung entschieden haben, geringer zu sein [26]. Die niedrigere Akzeptanz von EMHTools im klinischen Setting wirkte sich in einer Subgruppenanalyse jedoch nicht auf die Effektivität der Intervention aus [15].

In der Routineversorgung ist das Wissen um die Implementierung von Onlineinterventionen bei der Depressionsbehandlung noch gering [10]. Wirksamkeitsnachweise für EMH-Programme liegen für die ambulante Primärversorgung vor [20], wobei eine iCBT in der hausärztlichen Versorgung depressiver Erkrankungen keinen Zusatznutzen erbringt, wenn sie nur durch technischen Support und nicht durch einen Therapeuten begleitet wird [11]. Erste Wirksamkeitsbelege im stationären Kontext lieferte eine Untersuchung zur Kombination eines onlinebasierten Selbstmanagementprogrammes (oSMP) mit einer stationären Psychotherapie [30]. Die Follow-up-Untersuchung ergab bei Fortführung der Intervention im Anschluss an die stationäre Therapie eine höhere Lebensqualität sowie eine geringere depressive Residualsymptomatik
[29]. Weiterhin kann iCBT zu einer Erhöhung von Remissionsraten sowie zu einer Reduktion der Wahrscheinlichkeit eines Rezidives beitragen [13]. Allerdings wurden bei Onlineinterventionen hohe Dropout-Raten beobachtet [31]. Um diese zu reduzieren und die Komplettierungsrate von EMH-Interventionen zu erhöhen, eignet sich insbesondere die Steigerung der präinterventionellen Nutzungsakzeptanz [18].

Ein EMH-Tool ist das kostenfreie Onlineselbstmanagementprogramm „iFightDepression“ (iFD), welches sich aus verschiedenen Workshops konstituiert und auf Techniken der kognitiven Verhaltenstherapie basiert. Das iFD-Tool ist in der ambulanten Versorgung für leichte bis mittelschwere Depression vorgesehen, in dieser Studie wurden aufgrund des stationären Settings PatientInnen mit schwerer Depression inkludiert.

Vor dem Hintergrund der möglichen positiven Aspekte einer Nutzung von EMHProgrammen in der Depressionsbehandlung, aber dem geringen Wissen über ihren Einsatz im stationären Setting, war das Ziel unserer Studie, das iFD-Tool als zusätzliches Behandlungsangebot zur leitliniengerechten depressionsspezifischen stationären Behandlung zu implementieren und zu evaluieren.

\section{Methodik}

Die Studie wurde von Juni 2018 bis Februar 2020 auf der Spezialstation für Affektive Störungen der Klinik und Poliklinik für Psychiatrie und Psychotherapie des Universitätsklinikum Leipzig mit Zustimmung der zuständigen Ethikkommission der Medizinischen Fakultät der Universität Leipzig (AZ: 164/17-ek) durchgeführt.

\section{Studiendesign}

Die Teilnehmenden nutzten das iFD-Tool als Zusatzzum leitliniengerechten Behandlungskonzept der Station mittels eigener mobiler Endgeräte. Die Nutzung sollte in den therapiefreien Zeiten erfolgen. Die Interventionsdauer entsprach der Dauer der stationären Behandlung. Zu Interventionsbeginn (stationäre Aufnahme, T0) füllten die Teilnehmer einen Onlinefragebogen aus, bei Entlassung bearbeiteten die 
Hier steht eine Anzeige.

黑 Springer 
Tab. 1 Ein- und Ausschlusskriterien

\begin{tabular}{|l|l|}
\hline Einschlusskriterien & Ausschlusskriterien \\
\hline Unipolare Depression & Demenz \\
\hline Dysthymie & Schizophrenie \\
\hline Alter $\geq 18$ Jahre & Suchterkrankung \\
\hline Längerfristiger stationärer Aufenthalt & Bipolare Störung \\
\hline Gültige E-Mail-Adresse & Zwangsstörung \\
\hline Schriftliche und elektronische Einwilligung & Persönlichkeitsstörung (F60.2 oder F60.3) \\
\hline & Suizidalität \\
\hline & $\begin{array}{l}\text { Fehlende Medienkompetenz (subjektive Ein- } \\
\text { schätzung/keine eigene E-Mail-Adresse) }\end{array}$ \\
\hline
\end{tabular}

\begin{tabular}{|l|l|l|}
\hline Tab. 2 Workshops & KVT-basierter Inhalt & Intervention \\
\hline Workshop & $\begin{array}{l}\text { Denken, Fühlen und } \\
\text { Handeln }\end{array}$ & Aktivitätenprotokoll \\
\hline $\begin{array}{l}\text { Schlaf und Depres- } \\
\text { sion }\end{array}$ & $\begin{array}{l}\text { Schlafverhalten und depressive } \\
\text { Symptomatik }\end{array}$ & Schlaftagebuch \\
\hline $\begin{array}{l}\text { Schöne Dinge planen } \\
\text { und unternehmen }\end{array}$ & Tagesstrukturierung & Planung regelmäßiger Aktivitäten \\
\hline $\begin{array}{l}\text { Dinge erledigen } \\
\text { Negative Gedanken } \\
\text { erkennen }\end{array}$ & Problemlösetraining & $\begin{array}{l}\text { Antizipieren von Schwierigkeiten } \\
\text { und Lösungsmöglichkeiten anste- } \\
\text { hender Unternehmungen }\end{array}$ \\
\hline $\begin{array}{l}\text { Negative Gedanken } \\
\text { verändern }\end{array}$ & Kognitive Umstrukturierung & $\begin{array}{l}\text { Ereignisse, Gedanken, Reaktionen } \\
\text { reflektieren }\end{array}$ \\
\hline $\begin{array}{l}\text { Zusatz: ein gesunder } \\
\text { Lebensstil }\end{array}$ & $\begin{array}{l}\text { Psychoedukation: stabilisieren- } \\
\text { de Umgebungsfaktoren }\end{array}$ & \\
\hline KVT Kognitive Verhaltenstherapie; $A B C$ : $A$ Activating event, B Belief, C Consequences \\
\hline
\end{tabular}

PatientInnen einen Paper-pencil-Fragebogen (T1). Die iFD-Tool-Nutzung war auch nach der Entlassung möglich, eine Evaluation ab diesem Zeitpunkt erfolgte jedoch nicht mehr. Begleitet wurden sie vom Studienverantwortlichen, welcher ein Onlinetraining zu dem iFD-Tool durchlaufen hatte. Die Begleitung erfolgte einmal wöchentlich à 30-60 min im Rahmen einer Gruppentherapie mit Aufklärung und Anleitung zu den Inhalten der Intervention sowie Raum für Fragen und Feedback. Auch das gesamte Pflege- und ÄrztInnen/ PsychologInnen-Team durchlief das Onlinetrainingsprogramm zu dem iFD-Tool, damit sie Fragen der StudienteilnehmerInnen jederzeit auch außerhalb des wöchentlichen Meetings beantworten konnten. Nichtteilnehmende konnten freiwillig Ablehnungsgründe angeben.

\section{Stichprobe}

Während der Studiendauer wurden alle neu aufgenommenen und die Einschlusskriterien erfüllenden PatientInnen konsekutiv gefragt. Eingeschlossen wurden 78 PatientInnen (- Abb. 1), die unter einer unipolaren Depression unterschiedlichen Schweregrades (ICD-10 F32.0-3, F33.0-3) oder Dysthymie (F34.1) litten. Die Diagnosestellung erfolgte durch das ärztliche Stationspersonal. Ein- und Ausschlusskriterien sind in $\mathbf{0}$ Tab. 1 aufgeführt.

\section{Das iFD-Tool}

Das iFD-Tool ist ein kostenloses, therapeutenbegleitetes OSMP für Erwachsene und Jugendliche basierend auf kognitiver Verhaltenstherapie. Interessierte HausärztInnen, aber auch FachärztInnen für Psychiatrie und Psychotherapie sowie psychologische PsychotherapeutInnen können ein kostenloses Online- training (https://ifightdepression.com/ webinar/) durchlaufen und anschließend PatientInnen zur Nutzung freischalten. Das iFD-Tool enthält 6 Kernworkshops und einen Zusatzworkshop (• Tab. 2).

\section{Untersuchungsinstrumente}

Der T0-Fragebogen wurde als Bestandteil des iFD-Tools erhoben. Erfragt wurde die psychiatrische Vorgeschichte und die an den Kontext IMI adaptierte Subskala Allgemeine Behandlungserwartung (BE) des Fragebogens zur Messung der Psychotherapiemotivation (FMP) anhand von 8 Items [25]. Zwei weitere vom iFDEntwickler-Team konstruierte Items zur Einstellung bezüglich IMI, ein Item zum „shared decision-making" sowie das Alter und Geschlecht wurden ebenfalls erfasst.

Die Veränderung der depressiven Symptomatik wurde wöchentlich mit dem im iFD-Tool implementierten Patient Health Questionnaire 9 (PHQ-9, Summenwert: 0-27) erfasst [16].

Der T1-Fragebogen enthielt Fragen zu dem Bearbeitungsumfang, der Art und Zufriedenheit mit der Begleitung, der Nutzungsmodalität, der Interventionszufriedenheit (anhand des ZUF-8 [23]), der subjektiven Nützlichkeit des iFD-Tools (anhand des USE-Questionnaires [17]) sowie dem Vorhaben der weiteren Nutzung. Nutzungsdaten konnten über das iFD-Tool anonymisiert erfasst werden.

\section{Statistik}

Die statistische Analyse erfolgte deskriptiv mit IBM SPSS Statistics Version 22 (IBM Corp, Armonk, NY, USA). Der PHQ-9-Verlauf wurde mit einer Varianzanalyse bei abhängigen Stichproben analysiert. Die Gruppenunterschiede zum Vergleich der Behandlungserwartung und -zufriedenheit zwischen Frauen und Männern sowie dem Patientenalter $<39$ bzw. $>39$ Jahren wurden für normalverteilte, varianzhomogene Daten mittels des Zweistichproben-tTests analysiert, bei Varianzinhomogenität wurde der Welch-Test eingesetzt. Die Zusammenhangsanalyse erfolgte mittels bivariater Korrelationsanalyse. Ein zweiseitiges Signifikanzniveau von $\alpha=0,05$ wurde als bedeutsam betrachtet. 
Tab. 3 Demographische und klinische Charakteristika

\begin{tabular}{|c|c|c|}
\hline Variable & $\begin{array}{l}\text { Alle Studienteilnehmer- } \\
\text { Innen }(n=78)\end{array}$ & \\
\hline Geschlecht (weiblich), $n$ (\%) & $51(65,4)$ & \\
\hline \multirow[t]{2}{*}{ Alter (Jahre), M (SD) } & $39,9(13,2)$ & \\
\hline & Aktiv nutzend $(n=42)$ & $\begin{array}{l}\text { Nicht nutzend } \\
(n=36)\end{array}$ \\
\hline Geschlecht (weiblich), $n$ (\%) & $27(64,3)$ & $24(66,7)$ \\
\hline Alter (Jahre), M (SD) & $41,5(11,3)$ & $38,2(12,0)$ \\
\hline Depressive Episode in der Vergangenheit $n(\%)$ & $26(61,9)$ & \\
\hline \multicolumn{3}{|l|}{ Therapieerfahrung } \\
\hline Pharmakotherapie, $n$ (\%) & $37(88,1)$ & \\
\hline Psychotherapie, $n(\%)$ & $35(83,3)$ & \\
\hline Andere $^{\mathrm{a}}, n(\%)$ & $12(28,6)$ & \\
\hline $\begin{array}{l}\text { Median selbstberichteter depressiver Episoden in } \\
\text { der Vergangenheit, } n \text { (SW) }\end{array}$ & $3,0(1-8)$ & \\
\hline \multicolumn{3}{|c|}{$\begin{array}{l}\text { M Mittelwert, SD Standardabweichung, SW Spannweite } \\
\text { aAndere Therapieerfahrungen: Elektrokrampftherapie, Schlafentzug, ambulante Ergotherapie, ambu- } \\
\text { lante psychiatrische Pflege }\end{array}$} \\
\hline
\end{tabular}

\begin{tabular}{|l|l|}
\hline Tab. $4 \quad$ Itemauswertung & Median/MW (SD) \\
\hline Item & \\
\hline Behandlungserwartung & $3 / 3,3(0,98)$ \\
\hline 1 Mal ausspannen hilfreicher als oSMP & $2 / 2,5(1,1)$ \\
\hline 2 Nur medizinische Behandlung kann meine Beschwerden mindern & $4,5 / 4,2(0,91)$ \\
\hline 3 Kann noch vieles lernen, was hilft, meine Krankheit zu bewältigen & $3 / 2,8(1,0)$ \\
\hline 4 Lösung meiner Probleme hilfreicher als oSMP & $4 / 4,2(0,79)$ \\
\hline 5 Kann aktiv zur Beschwerdebesserung beitragen & $2 / 2,4(0,74)$ \\
\hline 6 Arzt wäre passender als oSMP & $4 / 3,7(0,81)$ \\
\hline 7 Glaube kaum, dass oSMP hilft & $3 / 3,2(1,1)$ \\
\hline 8 Medikament/Operation wären mir lieber als oSMP & \\
\hline Einstellung gegenüber IMI & $2 / 2,1(0,73)$ \\
\hline 9 Sehe Vorteile in der Nutzung von oSMP & $1 / 1,5(0,84)$ \\
\hline 10 Persönlicher Behandlerkontakt ist wichtig & \\
\hline Entscheidungsprozess & $1 / 1,6(0,91)$ \\
\hline 11 War an der Nutzungsentscheidung aktiv beteiligt & \\
\hline $\begin{array}{l}\text { oSMP onlinebasiertes Selbstmanagementprogramm, MW Mittelwert, SD Standardabweichung, } \\
\text { IMI internet- und mobilbasierte Intervention }\end{array}$ \\
\hline
\end{tabular}

\section{Ergebnisse}

\section{Beschreibung der Stichprobe}

Von den 78 Teilnehmenden loggten sich 42 mindestens einmal ein, 36 nutzten das iFD-Tool nicht. - Tab. 3 zeigt die demographischen und klinischen Charakteristika der Stichprobe.

\section{Pretreatment}

Daten zur Interventionserwartung liegen von 42 Personen vor, - Tab. 4 zeigt die Auswertung der Einzelitems. Unter-
Items 1, 2, 4, 6, 7, 8, 9, 10, 11 wurden von 1-5 bewertet, die Items 3 und 5 wurden invers skaliert. Die allgemeine Behandlungserwartung lag bei $M=26,23$ $(S D=3,25)$. Es wurde angenommen, dass es zwischen Geschlecht und Alter keine Differenzen bezüglich Erwartung und Zufriedenheit gibt. Im T-Test (0Tab. 7) unterschieden sich weder Frauen/Männer $\left(\mathrm{t}_{[39]}=-0,77, p=0,45\right)$ noch Patienten unter/über 39 Jahren $\left(\mathrm{t}_{[38]}=1,17, p=0,25\right)$ signifikant.

Der initiale PHQ-9-Wert zeigte keinen signifikanten Zusammenhang mit der Behandlungserwartung $(r=-0,19 ; p=0,29)$.

\section{Verlauf der Depressionsschwere}

Der Anteil schwergradig Erkrankter war mit $54,8 \%$ verglichen mit der Gesamtkohorte hoch (•Tab. 5). Im Mittel lag der initiale PHQ-9 Wert bei $M=14,93(S D=5,11)$ und fiel während des stationären Aufenthaltes von 14,93 auf 7,5 (-7,43; $95 \%$-Konfidenzintervall-8,14 bis $-6,73 ; p<0,05)$. Die Anzahl der ausgefüllten Bögen pro Woche nahm mit der Zeit ab, was eine abnehmende Nutzerrate im zeitlichen Verlauf impliziert, da der PHQ-9 wöchentlich obligat abgefragt wurde (• Abb. 2).

\section{Posttreatment}

Drei Teilnehmende hatten die Nutzung abgeschlossen, 21 hatten dies noch vor und 18 hatten die Nutzung unterbrochen, um sie bei Bedarf fortzusetzen. 29 PatientInnen hatten das iFD-Tool seltener als einmal pro Woche genutzt, 6 PatientInnen nutzten das iFD-Tool einmal pro Woche, 4 der Teilnehmenden gaben an, das iFDTool mehrmals wöchentlich genutzt zu haben. 2 Patientlnnen nutzten das iFD-Tool täglich und eine Person mehrmals täglich. Im Median wurde das iFD-Tool für $10 \mathrm{~min}$ und 3 Sessions genutzt. Die Interventionszufriedenheit lag bei $M=23,1(S D=4,76$, Summenwerte: 8-32). DTab. 6 zeigt die Auswertung der Einzelitems, diese wurden 4-stufig (1: geringe Merkmalsausprägung bis 4: hohe Merkmalsausprägung) erfasst und sind in prozentualer Zustimmung angegeben.

Gering fiel die Bewertung der subjektiven Nützlichkeit aus $(M=15,35, S D=5,96$, Summenwerte: 7-35), ebenso wie die 
Tab. 5 Schweregrad der initialen depressiven Symptomatik anhand des Patient Health Questionnaires 9 (PHQ-9) ${ }^{\mathrm{a}}$

\begin{tabular}{|c|c|}
\hline Minimal $n(\%)$ & $1(2,3)$ \\
\hline Mild $n(\%)$ & $5(11,9)$ \\
\hline Mittelgradig $n(\%)$ & $13(31,0)$ \\
\hline Schwer $n(\%)$ & $23(54,8)$ \\
\hline \multicolumn{2}{|c|}{$\begin{array}{l}\text { Der Summenwert des PHQ-9 lässt } \\
\text { sich in } 4 \text { Kategorien zur Abbildung der } \\
\text { Stärke der depressiven Symptomatik } \\
\text { einteilen: } 0-4=\text { minimal; } 5-9=\text { mild; } \\
\text { 10-14= mittelgradig; } 15-27=\text { schwer }\end{array}$} \\
\hline
\end{tabular}

Tab. 6 Interventionszufriedenheit

Item

\begin{tabular}{|l|l|}
\hline Wie beurteilen Sie die Qualität von iFD? & Zustimmung (\%) \\
\hline Haben Sie mit iFD die Art von Unterstützung erhalten, die Sie wollten? & 61,4 \\
\hline Hat iFD Ihren Bedürfnissen entsprochen? & 58,1 \\
\hline Würden Sie das iFD-Tool empfehlen? & 63,7 \\
\hline Wie zufrieden sind Sie mit dem Ausmaß der Hilfe? & 60,4 \\
\hline Hat das Tool geholfen, angemessener mit Problemen umzugehen? & 60,4 \\
\hline Wie zufrieden sind Sie mit dem Tool? & 64,3 \\
\hline Würden Sie das Tool wieder benutzen? & 60,4 \\
\hline iFD „iFightDepression“ &
\end{tabular}

Tab. 7 Ergebnisse zur Behandlungserwartung und -zufriedenheit bezogen auf das iFD(„,FightDepression“)-Tool

\begin{tabular}{|l|l|l|}
\hline & $\begin{array}{l}\text { Behandlungserwartung } \\
\mathbf{M}(\text { SD) }\end{array}$ & $\begin{array}{l}\text { Behandlungszufriedenheit } \\
\mathbf{M}(\text { SD) }\end{array}$ \\
\hline Frauen & $26,0(3,16)$ & $22,2(3,20)$ \\
\hline Männer & $26,8(3,53)$ & $23,4(3,10)$ \\
\hline Alter $>39$ Jahre & $26,9(2,26)$ & $22,8(3,26)$ \\
\hline Alter <39 Jahre & $25,8(3,83)$ & $22,4(3,18)$ \\
\hline SD Standardabweichung, $M$ Median \\
\hline
\end{tabular}

2 Items der Skala "usefulness" $(M=6,61$, $S D=2,44$, Summenwerte: 2-14). 28 Teilnehmende gaben an, das iFD-Tool poststationär weiter nutzen zu wollen. Hauptgründe gegen die Fortführung waren geringe Medienaffinität, fehlendes Therapeutenfeedback, ausbleibender Therapieeffekt und hoher Zeitaufwand.

\section{Behandlungszufriedenheit}

Zwischen den Geschlechtern $\left(t_{[28]}=0,94\right.$, $p=0,35)$ und dem Alter $\left(\mathrm{t}_{[28]}=-0,31\right.$, $p=0,76)$ gab es keinen signifikanten Unterschied der Behandlungszufriedenheit in Bezug auf das iFD-Tool (• Tab. 7).

\section{Diskussion}

Das Ziel unserer Studie war, den Nutzen und die Anwendbarkeit des iFD-Tools für depressive PatientInnen im stationären Setting zu untersuchen. $62 \%$ (48/78) der Studienteilnehmenden registrierten sich zur Nutzung des iFD-Tools. Insgesamt nutzten $54 \%$ (42/78) der TeilnehmerInnen das Angebot mindestens einmal während ihres Aufenthaltes, aber nur $17 \%$ (13/78) der PatientInnen nutzten das iFDTool ein- oder mehrmals pro Woche. Ähnlich geringe Nutzungsraten zeigen andere Studien zu IMI im stationären [9,
31] und ambulanten Setting [20]. Verglichen mit dem notwendigen Aufwand für die Toolimplementierung, welche Personalschulungen und eine regelmäßige Betreuung der NutzerInnen erforderte, ist die Nutzungsrate im stationären Setting aktuell als eher gering zu bewerten. Im Rahmen zunehmender Digitalisierungsprozesse und der pandemiebedingten Einschränkungen psychiatrischer Behandlungsangebote bleibt abzuwarten, ob IMI in Zukunft eine erhöhte Nachfrage und Nutzungsbereitschaft, auch im stationären Sektor, erfährt.

\section{Für wen ist das iFD-Tool geeignet?}

Barrieren für die Nutzung waren eine kurze Dauer des stationären Aufenthaltes, fehlende subjektive Medienkompetenz und eine schwere depressive Symptomatik. Als Hauptgründe für die Nichtnutzung des iFD-Tools trotz Studienteilnahme wurden Zeitmangel aufgrund anderer Faceto-face-Therapieangebote, der Schweregrad der Depression und die Länge der Texte seitens der Patienten angegeben. Als akzeptanz- und compliancefördernd hat sich die Demonstration des iFD-Tools und der technische Support durch das Studienteam im Rahmen einer eigens hierfür etablierten Gruppentherapie er- wiesen. Diese Erfahrung deckt sich mit der besseren Wirksamkeit von begleiteten IMI [14] und der Steigerung der Akzeptanz durch den Erhalt expertengestützter Informationen und Empfehlungen [4].

Die Werte des PHQ-9 nahmen im Verlauf der stationären Therapie sukzessive ab. Aufgrund der multimodalen Behandlung und dem abnehmenden Fragebogenrücklauf lässt sich dies jedoch nicht unbedingt alleinig auf das iFD-Tool zurückführen.

Mit 26,23 von 40 Punkten fiel der Erwartungswert leicht überdurchschnittlich aus, ähnlich zu Untersuchungen von IMI im ambulanten Setting [6, 8]. Die Teilnehmenden signalisierten im PretreatmentFragebogen Lern- und Handlungsbereitschaft bezüglich der Intervention.

\section{Aspekte der Nutzererfahrung im stationären Setting}

Die PatientInnen präferierten eine Faceto-face-Therapie, was im Rahmen des klinischen Settings nachvollziehbar ist. Die PatientInnen sahen sich als aktiv entscheidend im Nutzungsprozess an, erkennbar an der hohen Zustimmung zur Einnahme einer aktiven Rolle im Entscheidungsprozess (-Tab. 4, Item 11), was für eine Fortführung der Nutzung relevant ist und in einer Untersuchung der IMI "color your life" [8] ähnlich evaluiert wurde.

Die moderat positive Interventionszufriedenheit deckt sich mit den Angaben zur iFD-Tool-Nutzung im ambulanten Setting [21]. Im stationären Rahmen erzielte "moodgym" eine ähnliche Nutzerzufriedenheit [9], ebenso andere EMH-Interventionen [2, 12]. Möglicherweise steigen die Zufriedenheitswerte, wenn die Inhalte solcher Tools mit den weiteren stationären Therapieangeboten besser abge- 


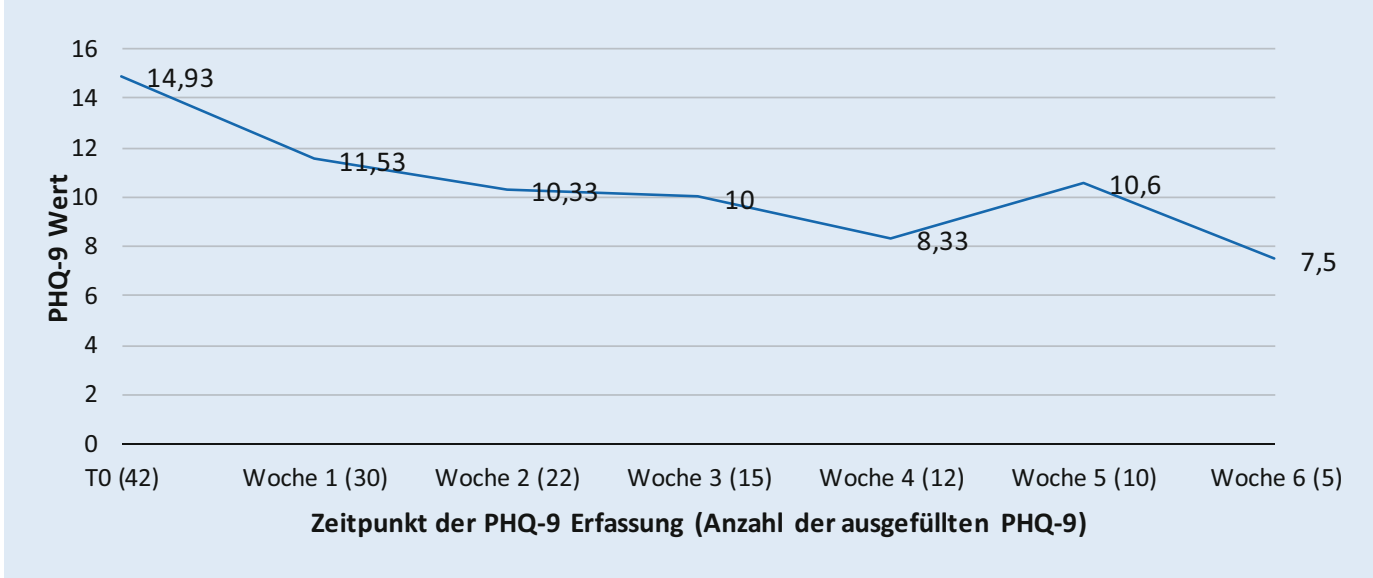

Abb. $2 \triangleleft$ Mittelwert Patient Health Questionnaire 9 (PHQ-9) im Verlauf des stationären Aufenthalts

stimmt werden [5], was sich im Rahmen des "blended-treatment", also der Kombination aus Face-to-face-Psychotherapie und einer Onlineintervention, als wirksam erwiesen hat [30].

Eine geringe Komplettierungsrate der Onlineintervention findet sich ebenso in anderen EMH-Bereichen [28]. Dies könnte generell ein Hinweis für die Notwendigkeit einer bedarfsgerechten Anpassung solcher Tools sein.

Die Nützlichkeit wurde als gering beurteilt, wohingegen die Auswertung jedes Workshops einzeln ergab, dass 38 Personen einen oder mehrere Workshops als hilfreich ansahen, während nur 4 Teilnehmer einen oder mehrere Workshops als nicht hilfreich oder gar belastend empfanden.

Trotz der moderat bewerteten Gesamtnützlichkeit gaben $67 \%$ der aktiven Nutzer an, das iFD-Tool poststationär weiter nut- zen zu wollen, was aufgrund der fehlenden Nachverfolgung nicht überprüft werden konnte. Die Möglichkeit der langfristigen Nutzung ist ein relevanter Aspekt und kann zur nachhaltigen Symptomreduktion beitragen [29], sollte aber in weiteren Studien untersucht werden.

Limitationen der Ergebnisse dieser Studie sind die geringen Fallzahlen und die fehlende Kontrolle möglicher Medikamenten- und Psychotherapieeinflüsse. Des Weiteren ergaben sich aufgrund des naturalistischen Studiendesigns unterschiedlich lange Anwendungszeiten, welche die Behandlungsrealität eines stationären Settings abbilden, allerdings auch die Vergleichbarkeit erschweren.

Die Limitationen ergeben sich teilweise aufgrund der Wahl eines naturalistischen Settings, welches eine praxisnahe Evaluierung des Tools im klinischen Alltag ermöglichte. Die Anwendung auch bei schwerer depressiver Symptomatik führte einerseits zu einer symptombedingten Einschränkung der Anwendbarkeit seitens der Nutzer, ermöglichte aber andererseits auch erste Erfahrungen mit einer Nutzung des iFD-Tools bei schwerer depressiver Episode.

\section{Fazit für die Praxis}

- Die Implementierung des iFD-Tools bei depressiver Erkrankung erwies sich im stationären Rahmen als machbar und führte bei Nutzung zu vorwiegend positivem Feedback seitens der PatientInnen, ergab jedoch insgesamt geringe Nutzungsraten.

- Barrieren für die Anwendung im stationären Setting waren eine kurze Dauer des stationären Aufenthalts, Zeitmangel aufgrund anderer Face-to-face-Therapieangebote, eine besonders schwere depressive Symptomatik und eine fehlende Medienkompetenz der PatientInnen.

\section{Hier steht eine Anzeige.}

\section{望 Springer}


- Eine gezielte Ansprache medienaffiner Personen erhöht die Wahrscheinlichkeit einer Nutzung des iFD-Tools. Um die Compliance zu erhöhen, sollte die Intervention therapeutisch begleitet werden. Dies kann im stationären Setting auch als Gruppenformat erfolgen.

- Eine poststationäre Fortführung der Nutzung wurde von den meisten aktiven Nutzern gewünscht. Dies kann im Entlassungsmanagement integriert werden und sollte im Rahmen zukünftiger Studien untersucht werden.

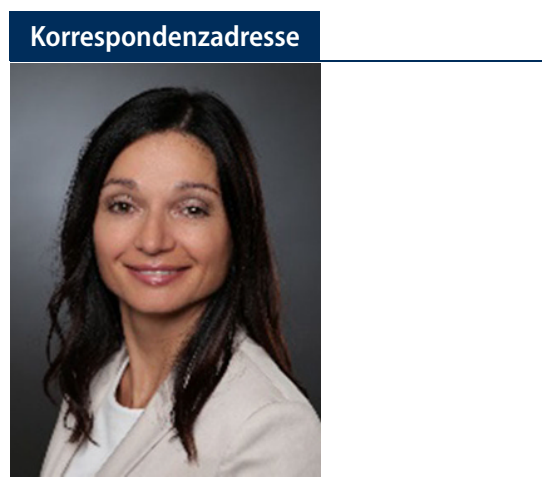

PD Dr. med. Maria Strauß, MHBA

Department für Psychische Gesundheit, Klinik und Poliklinik für Psychiatrie und Psychotherapie, Universitätsklinikum Leipzig, AöR

Semmelweisstr. 10, 04103 Leipzig, Deutschland maria.strauss@medizin.uni-leipzig.de

Funding. Open Access funding enabled and organized by Projekt DEAL.

\section{Einhaltung ethischer Richtlinien}

Interessenkonflikt. J.Schwarz, N. Mauche, C. Oehler, C. Rummel-Kluge, U. Hegerl und M. Strauß geben an, dass kein Interessenkonflikt besteht.

Für diesen Beitrag wurden von den Autoren keine Studien an Menschen oder Tieren durchgeführt. Für die aufgeführten Studien gelten die jeweils dort angegebenen ethischen Richtlinien.

Open Access. Dieser Artikel wird unter der Creative Commons Namensnennung 4.0 International Lizenz veröffentlicht, welche die Nutzung, Vervielfältigung, Bearbeitung, Verbreitung und Wiedergabe in jeglichem Medium und Format erlaubt, sofern Sie den/die ursprünglichen Autor(en) und die Quelle ordnungsgemäß nennen, einen Link zur Creative Commons Lizenz beifügen und angeben, ob Änderungen vorgenommen wurden.

Die in diesem Artikel enthaltenen Bilder und sonstiges Drittmaterial unterliegen ebenfalls der genannten Creative Commons Lizenz, sofern sich aus der Abbildungslegende nichts anderes ergibt. Sofern das betreffende Material nicht unter der genannten Creative

"iFightDepression" in inpatient treatment. Evaluation of a web-based, therapist-guided self-management program on a specialized ward for affective disorders

Background: E-mental health mainly plays a role in the outpatient treatment of patients with depressive disorders. The goal of this study was to implement and evaluate the web-based, therapist-guided self-management tool "iFightDepression" (iFD) to clarify if there is a benefit for inpatient use.

Material and methods: In this study 78 inpatients with affective disorders (ICD-10 F32.0-3, F33.0-3) or dysthymia (F34) were recruited. The intervention duration with the iFD tool went from admission until discharge, therapeutic support was granted by the ward staff. Symptom severity, intervention expectations and experience with therapy were processed in an online questionnaire before the intervention (T0) while intervention satisfaction was captured after the intervention shortly before discharge (T1) in a paper-pencil questionnaire.

Results: Out of 78 participating inpatients 42 used the iFD tool at least once. Moderate to high levels of expectation regarding the iFD tool and mildly above-average level of satisfaction after the intervention were observed. Of the active users $67 \%$ indicated they would continue to use the iFD tool after discharge. The main reasons for not using the iFD tool were short duration of stay, severity of disease and lack of digital literacy. Conclusion: An implementation of the iFD tool is feasible on principle. Active users gave positive feedback concerning the intervention and most participants claimed to continue using the iFD tool after discharge; however, low usage rates among study participants $(42 / 78,54 \%)$ showed barriers of implementation that have to be addressed and underline the importance of adaptations regarding the use of the intervention in a clinical setting.

\section{Keywords}

E-mental health - Depression · Cognitive behavioral therapy - Self-management · Clinical study

Commons Lizenz steht und die betreffende Handlung nicht nach gesetzlichen Vorschriften erlaubt ist, ist für die oben aufgeführten Weiterverwendungen des $\mathrm{Ma}$ terials die Einwilligung des jeweiligen Rechteinhabers einzuholen.

Weitere Details zur Lizenz entnehmen Sie bitte der Lizenzinformation auf http://creativecommons.org/ licenses/by/4.0/deed.de.

\section{Literatur}

1. Andersson $\mathrm{G}$ et al (2019) Internet interventions for adults with anxiety and mood disorders: a narrative umbrella review of recent meta-analyses. Can J Psychiatry 64(7):465-470

2. Andrews G et al (2018) Computer therapy for the anxiety and depression disorders is effective, acceptable and practical health care: an updated meta-analysis. J Anxiety Disord 55:70-78

3. Apolinário-Hagen Jetal (2017) Publicacceptability of e-mental health treatment services for psychological problems: a scoping review. JMIR Ment Health 4(2):e10

4. Apolinário-Hagen J et al (2018) Improving attitudes toward e-mental health services in the general population via psychoeducational information material: a randomized controlled trial. Internet Interv 12:141-149

5. Baumeister H et al (2018) Blended Psychotherapyverzahnte Psychotherapie: Das Beste aus zwei Welten? Psychother Dialog 04(19):33-38
6. Botella C et al (2016) An Internet-based program for depression using activity and physiological sensors: efficacy, expectations, satisfaction, and ease of use. Neuropsychiatr Dis Treat 12:393-406

7. Busch MA et al (2013) Prävalenz von depressiver Symptomatik und diagnostizierter Depression bei Erwachsenen in Deutschland: Ergebnisse der Studie zur Gesundheit Erwachsener in Deutschland (DEGS1). Bundesgesundheitsblatt Gesundheitsforschung Gesundheitsschutz 56(5-6):733-739

8. De Graaf LE et al (2009) Use and acceptability of unsupported online computerized cognitive behavioral therapy for depression and associations with clinical outcome. J Affect Disord 116(3):227-231

9. Dorow M et al (2018) Der komplementäre Einsatz des internetbasierten Selbstmanagementprogramms ,moodgym' bei Menschen mit depressiven Erkrankungen in der stationären Versorgung - die Perspektive von Patienten und Behandlern. Psychiat Prax 45(05):256-262

10. DrozdFetal (2016) The implementation of internet interventions for depression: a scoping review. JMed Internet Res 18(9):e236

11. Gilbody $S$ et al (2015) Computerised cognitive behaviour therapy (cCBT) as treatment for depression in primary care (REEACT trial): large scale pragmatic randomised controlled trial. BMJ 351:h5627

12. Shehadeh H et al (2020) Step-by-Step, an e-mental health intervention for depression: a mixed methods pilot study from Lebanon. Front Psychiatry 10:986 
13. Holländare F et al (2013) Two-year outcome of internet-based relapse prevention for partially remitted depression. Behav Res Ther 51(11):719-722

14. Karyotaki E et al (2021) Internet-based cognitive behavioral therapy for depression: a systematic review and individual patient data network metaanalysis. JAMA Psychiatry 4(78):361-371

15. Klein JP et al (2017) Does recruitment source moderate treatment effectiveness? A subgroup analysis from the EVIDENT study, a randomised controlled trial of an internet intervention for depressive symptoms. BMJ Open 7(7):e15391

16. Löwe B et al (2002) Gesundheitsfragebogen für Patienten (PHQ-D), 2. Aufl. Komplettversion und Kurzform. Testmappe mit Manual, Fragebögen, Schablonen

17. Lund AM (2001) Measuring usability with the use questionnaire. Usability Interface 8(2):3-6

18. Mira A et al (2019) Exploring the relationship between the acceptability of an Internet-based intervention for depression in primary care and clinical outcomes: secondary analysis of a randomized controlled trial. Front Psychiatry 10:325

19. Moessner M et al (2017) E-Mental-Health und internetbasierte Psychotherapie. Psychotherapeut 62(3):266-251

20. Newby JM et al (2014) Effectiveness of transdiagnostic internet cognitive behavioural treatment for mixed anxiety and depression in primary care. J Affect Disord 165:45-52

21. OehlerCetal (2020) Efficacy of a guided web-based self-management intervention for depression or dysthymia: randomized controlled trial with a 12month follow-up using an active control condition J Med Internet Res 22(7):e15361

22. Richards D et al (2018) Supported internetdelivered cognitive behavior treatment for adults with severe depressive symptoms: a secondary analysis. JMIRMent Health 5(4):e10204

23. Schmidt J et al (1989) Zufriedenheit mit der stationären Versorgung. Entwicklung eines Fragebogens und erste Validitätsuntersuchungen. Psychother Psychosom Med Psychol 39(7):248-255

24. Schneider F et al (2004) Insufficient depression treatment in outpatient settings. Ger Med Sci 2:Doc1

25. Schneider W et al (1989) Fragebogen zur Messung der Psychotherapie-Motivation: FMP. Beltz, Weinheim

26. Schröder J et al (2017) Attitudes towards internet interventions among psychotherapists and individuals with mild to moderate depression symptoms. Cogn Ther Res 41(5):745-756

27. Sztein DM et al (2018) Efficacy of cognitive behavioural therapy delivered over the Internet for depressive symptoms: a systematic review and meta-analysis. J Telemed Telecare 24(8):527-539

28. Wallin E et al (2018) Treatment activity, user satisfaction, and experienced usability of internetbased cognitive behavioral therapy for adults with depression and anxiety after a myocardial infarction: mixed-methods study. J Med Internet Res 20(3):e87

29. Zwerenz R et al (2019) Improving the course of depressive symptoms after inpatient psychotherapy using adjunct web-based self-help: followup results of a randomized controlled trial. J Med Internet Res 21(10):e13655

30. Zwerenz R et al (2017) Online self-help as an add-on to inpatient psychotherapy: efficacy of a new blended treatment approach. Psychother Psychosom 86(6):341-350
31. Zwerenz Ret al (2017) Transdiagnostic, psychodynamic web-based self-help intervention following inpatient psychotherapy: results of a feasibility study and randomized controlled trial. JMIR Ment Health 4(4):e41

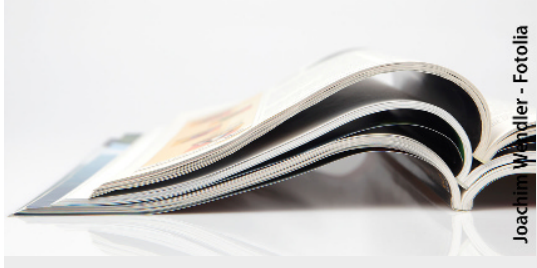

Haben Sie anderes Papier bei Ihrer Zeitschrift bemerkt?

Eine Mitteilung von Springer Nature / Springer Medizin

Wie viele andere Industriezweige hatte auch die Papierherstellung in den letzten Monaten mit Problemen in der Lieferkette zu kämpfen. Darüber hinaus stellten einige Papierfabriken von der Herstellung von Druckpapier auf die profitableren Verpackungsmaterialien um, insbesondere Karton wegen des boomenden OnlineHandels. Das verfügbare Papiervolumen ging Anfang dieses Jahres weiter zurück, da mehrere große Papierfabriken, darunter auch eine, auf die Springer Nature angewiesen ist, wegen eines Streiks geschlossen wurden.

\section{Papierknappheit wird anhalten}

Der Krieg in der Ukraine hat die ohnehin schon angespannte Lage auf dem globalen Papiermarkt, insbesondere in Europa, noch verschärft. Embargos für Holzexporte und Energielieferungen aus Russland werden die Papierknappheit weiter befeuern.

\section{Druckbetrieb bleibt unverändert}

In diesem Umfeld setzt Springer Nature seinen Druckbetrieb unverändert fort, ist aber mit Beschaffungsschwierigkeiten konfrontiert, die eine Flexibilität bei der Papiersorte für jede einzelne Zeitschrift erfordern. Wenn Sie also anderes Papier als bisher bei Ihrer Zeitschrift entdecken, so kennen Sie jetzt den Hintergrund. Springer Nature wird die gewohnten Sorten liefern, wenn der Papiermarkt dies zulässt. Wir bitten um Ihr Verständnis. 